\title{
Magnetic, Transport, and Phonon Properties of the Trivalent Eu Metallic Compound $\mathrm{EuBe}_{13}$
}

\author{
Hiroyuki Hidaka*, Kota Mizuuchi, Tatsuya Yanagisawa, and Hiroshi Amitsuka \\ Graduate School of Science, Hokkaido University, Sapporo, Hokkaido 060-0810, Japan
}

\begin{abstract}
Magnetic susceptibility $\chi$, specific heat $C$, and electrical resistivity $\rho$ measurements have been performed on singlecrystal $\mathrm{EuBe}_{13}$ in the temperature range between 2 and $300 \mathrm{~K}$ to investigate its phonon property and the valence state of the Eu ion. The obtained $\chi(T)$ curves obey a typical Van Vleck susceptibility for $\mathrm{Eu}^{3+}$ with a nonmagnetic ground state in the entire measured temperature range. In the case of $C(T)$, we observed the coexistence of Debye and Einstein phonon modes with characteristic Debye and Einstein temperatures of $\theta_{\mathrm{D}} \sim 835 \mathrm{~K}$ and $\theta_{\mathrm{E}} \sim 167 \mathrm{~K}$, respectively, which are in good agreement with those previously reported for other isostructural $\mathrm{MBe}_{13}$ compounds $(\mathrm{M}=$ rare earths and actinides). The $\rho(T)$ curve for $\mathrm{EuBe}_{13}$ shows an unusual $T^{3}$-like dependence at low temperatures, as also observed for the nonmagnetic isostructural compound $\mathrm{LaBe}_{13}$, which can be reproduced well by calculations based on electron-phonon scattering using the estimated $\theta_{\mathrm{D}}$ and $\theta_{\mathrm{E}}$. We also summarized the relationship between the Eu-valence state and the free distance between the Eu ion and the first-nearest-neighbor atoms in several Eu-based cubic compounds, and argued that $\mathrm{EuBe}_{13}$ takes the $\mathrm{Eu}^{3+}$ state despite its larger free distance than other $\mathrm{Eu}^{3+}$ compounds.
\end{abstract}

\section{Introduction}

$\mathrm{MBe}_{13}$ compounds $(\mathrm{M}=$ rare earths and actinides) show a rich variety of physical properties depending on the $M$ ion, such as unconventional superconductivity (SC) and nonFermi-liquid behavior in $\mathrm{UBe}_{13},{ }^{1,2)}$ an intermediate valence state in $\mathrm{CeBe}_{13},{ }^{3)}$ helical magnetic ordering in $\mathrm{HoBe}_{13},{ }^{4)}$ and nuclear antiferromagnetic (AFM) ordering in $\operatorname{PrBe}_{13} .{ }^{5)}$ They crystallize in a $\mathrm{NaZn}_{13}$-type cubic structure with the space group $F m \overline{3} c$ (No. 226, $O_{h}^{6}$ ), where the unit cell contains M atoms in the $8 a$ site, $\mathrm{Be}^{\mathrm{I}}$ atoms in the $8 b$ site, and $\mathrm{Be}^{\mathrm{II}}$ atoms in the $96 i$ site. ${ }^{6-8)}$ It is notable that the $\mathrm{MBe}_{13}$ compounds can be categorized as cage-structured compounds, since the unit cell consists of two cagelike structures; the $\mathrm{M}$ atom is surrounded by $24 \mathrm{Be}^{\mathrm{II}}$ atoms, nearly forming a snub cube, and the $\mathrm{Be}^{\mathrm{I}}$ atom is surrounded by $12 \mathrm{Be}^{\mathrm{II}}$ atoms, forming an icosahedron cage. Such cage-structured compounds have attracted much attention because of the presence of a low-energy phonon mode associated with local vibration of a guest atom with a large amplitude in an oversized host cage, so-called rattling. ${ }^{9-14)}$ The low-energy phonon mode has been considered to be related to several intriguing phenomena, such as rattlinginduced superconductivity ${ }^{15}$ and a magnetic-field-insensitive heavy-fermion state, ${ }^{16,17)}$ via electron-phonon coupling.

In several $\mathrm{MBe}_{13}$ compounds, such as $\mathrm{LaBe}_{13}, \mathrm{SmBe}_{13}$, $\mathrm{UBe}_{13}$, and $\mathrm{ThBe}_{13}$, a low-energy phonon mode, which can be described well by a model assuming a conventional harmonic Einstein phonon, has also been observed. ${ }^{18-20)}$ These findings suggest that the low-energy phonon mode is common to the $\mathrm{MBe}_{13}$ compounds. Previous results of inelastic neutron scattering (INS) and powder X-ray diffraction (XRD) measurements strongly indicate that the $\mathrm{M}$ atom behaves as an Einstein oscillator with characteristic temperature $\theta_{\mathrm{E}} \sim 160$ $\mathrm{K}$, whereas the $\mathrm{Be}$ atoms form the crystal lattice described by the Debye model with characteristic temperatures $\theta_{\mathrm{D}} \sim 600$ $-800 \mathrm{~K} .^{18,20,21)}$ Interestingly, the obtained $\theta_{\mathrm{E}}$ values in these systems appear to be independent of either the mass of the guest atoms or the guest free distances in the snub cube, which is a characteristic feature not found in other cage-structured compounds having a similar low-energy phonon mode. ${ }^{11-14)}$ To obtain further insight into the characteristics of the lowenergy phonon modes and their effects on the electronic states in $\mathrm{MBe}_{13}$, it is necessary to explore the phonon and electronic properties in other isostructural $\mathrm{MBe}_{13}$ compounds.

On the other hand, Eu-based compounds show two types of Eu valency: divalent $\left(\mathrm{Eu}^{2+}\right)$ and trivalent $\left(\mathrm{Eu}^{3+}\right)$. The ground state for $\mathrm{Eu}^{2+}$ is magnetic $\left(4 f^{7}: S=7 / 2, L=0\right.$, and $J=7 / 2$ ), while $\mathrm{Eu}^{3+}$ is nonmagnetic $\left(4 f^{6}: S=3, L=3\right.$, and $\left.J=0\right)$. Here, $S, L$, and $J$ are the total spin, total orbital, and total angular momenta, respectively. It is interesting that the number of intermetallic compounds with $\mathrm{Eu}^{3+}$ found thus far at ambient pressure is much smaller than that with $\mathrm{Eu}^{2+}$ or the intermediate valence state, ${ }^{22)}$ even though most rare-earth ions are usually trivalent in their compounds. The valence state of the Eu ion can be tuned easily by external parameters, such as temperature and pressure, because the energy difference between the two valence states is relatively small. ${ }^{23)}$ In this context, it will be useful to examine the relationship between the valence state and free space of the Eu ion in various Eu-based compounds, since the effective radii of Eu ions are different between the two valence states.

$\mathrm{EuBe}_{13}$ is a valuable material for studying not only the phonon property in $\mathrm{MBe}_{13}$ systems but also characteristics of the $\mathrm{Eu}^{3+}$ state. Its Eu valence has been revealed to be trivalent from previous magnetic susceptibility $(\chi)$ and Mössbauer spectroscopy measurements. ${ }^{6,24)}$ However, these measurements were performed for polycrystalline samples, and no information about the low-energy phonon mode and fundamental physical properties except for $\chi$ was given. ${ }^{6,24,25)}$ In this paper, we report the results of $\chi$, specific heat $(C)$, and electrical resistivity $(\rho)$ measurements on single-crystal $\mathrm{EuBe}_{13}$, and provide evidence of the presence of the low-energy phonon mode and the pure trivalent state of the Eu ion. 


\section{Experimental Procedure}

Single crystals of $\mathrm{EuBe}_{13}$ were grown by the Al-flux method. The constituent materials (Eu with $99.9 \%$ purity and Be with $99.9 \%$ purity) and Al with $99.99 \%$ purity were placed in an $\mathrm{Al}_{2} \mathrm{O}_{3}$ crucible at an atomic ratio of 1:13:35 and sealed in a quartz tube filled with Ar gas of $\sim 150 \mathrm{mmHg}$. The sealed tube was kept at $1050{ }^{\circ} \mathrm{C}$ for 3 days and then cooled at a rate of $2{ }^{\circ} \mathrm{C} / \mathrm{h}$. The $\mathrm{Al}$ flux was spun in a centrifuge and then removed using $\mathrm{NaOH}$ solution. The typical size of a grown sample is about $1 \times 1 \times 1 \mathrm{~mm}^{3}$. The results of powder XRD measurement at room temperature showed no impurity phase within the experimental accuracy except for reflections from a copper holder, although $\chi$ measurements indicate that the present single crystals include a minute amount of magnetic impurities, which may come from some Eu-Al binary alloy. A lattice parameter of $\mathrm{EuBe}_{13}$ was obtained to be $a=10.299(1) \AA$, which is close to the previously reported value of $a=10.286$ A. ${ }^{\text {) }}$

The DC magnetization $(M)$ was measured in the temperature range from 2 to $300 \mathrm{~K}$ at magnetic fields $B=0.1$ and 1 T using a Magnetic Property Measurement System (MPMS, Quantum Design, Inc.) and two crystal pieces (samples \#1 and \#2) taken from the same batch. $C$ was measured in the temperature range of $2-300 \mathrm{~K}$ at $0 \mathrm{~T}$ with a Physical Property Measurement System (PPMS, Quantum Design, Inc.) using a crystal piece (sample \#3) taken from the same batch. $\rho$ was measured using sample \#1 by a conventional four-probe method in the temperature range of $1.3-300 \mathrm{~K}$ at $0 \mathrm{~T}$ with a ${ }^{4} \mathrm{He}$ refrigerator. The electrical current $I$ was applied along the [100] direction.

\section{Experimental Results}

\subsection{Magnetic susceptibility}

Figure 1 shows the temperature dependence of the magnetic susceptibility $\chi(T)(=M(T) / B)$ for sample \#1 of EuBe $\mathrm{Eu}_{13}$ measured at $B=0.1$ and $1 \mathrm{~T}$ between 2 and $300 \mathrm{~K}$. The magnetic field was applied along the [100] axis. Both $\chi(T)$ curves gradually increase with decreasing temperature, and then become nearly constant below $\sim 100 \mathrm{~K}$. Below $\sim 50 \mathrm{~K}$, the $\chi(T)$ curves start to increase again and show a clear cusp at $\sim 15 \mathrm{~K}$ for $B=0.1 \mathrm{~T}$, while a broad shoulder appears for $B=1 \mathrm{~T}$. The increase in $\chi(T)$ at the low temperatures can also be observed in $\chi(T)$ at $0.1 \mathrm{~T}$ for sample \#2, as shown in the inset of Fig. 1, which is more prominent than that for sample \#1. These magnetic field and sample dependences indicate that the increase in $\chi(T)$ at low temperatures can be attributed to some magnetic impurities undetected in the XRD measurements. One of the possible impurities is the antiferromagnet $\mathrm{EuAl}_{4}$ with $\mathrm{Eu}^{2+}\left(T_{\mathrm{N}}=15.4 \mathrm{~K}\right),{ }^{26)}$ since the present $\chi(T)$ curves at $0.1 \mathrm{~T}$ show a cusp anomaly at $\sim 15 \mathrm{~K}$. In addition, a further upturn below $10 \mathrm{~K}$ in $\chi(T)$, which cannot be explained by $\mathrm{EuAl}_{4}$, indicates the presence of other magnetic impurities. We roughly estimated the amount of impurities in sample \#2 to be about $0.5 \%$, on the assumption that the low- $T$ increase in $\chi(T)$ at $0.1 \mathrm{~T}$ comes from $\mathrm{EuAl}_{4}$ and some other $\mathrm{Eu}^{2+}$ paramagnetic material.

The obtained $\chi(T)$ for $E_{13 B}$ well obeys the Van Vleck paramagnetic susceptibility, except for the low- $T$ increase due to the minute amount of magnetic impurities. In the case of $\mathrm{Eu}^{3+}$, the ground-state $J$ multiplet is $J=0$, and the energy of the excited $J$ multiplet $E_{J}$ is given as

$$
E_{J}=\frac{\lambda}{2} J(J+1),
$$

where $\lambda$ is the coupling constant of the spin-orbit interaction $\lambda \boldsymbol{L} \cdot \boldsymbol{S}$. The Van Vleck magnetic susceptibility $\chi_{\mathrm{vv}}$ can be expressed as: ${ }^{27)}$

$$
\chi_{\mathrm{vv}}(T)=\frac{\sum_{J=0}^{J=6} \chi_{J}(2 J+1) e^{-E_{J} / k_{\mathrm{B}} T}}{\sum_{J=0}^{J=6}(2 J+1) e^{-E_{J} / k_{\mathrm{B}} T}},
$$

where $\chi_{J}$ is written as

$$
\begin{gathered}
\chi_{J}=\frac{N_{\mathrm{A}} g_{J}^{2} \mu_{\mathrm{B}}^{2} J(J+1)}{3 k_{\mathrm{B}} T}+\alpha_{J}, \\
\alpha_{J}=\frac{N_{\mathrm{A}} \mu_{\mathrm{B}}^{2}}{6(2 J+1)}\left(\frac{F_{J+1}}{E_{J+1}-E_{J}}-\frac{F_{J}}{E_{J}-E_{J-1}}\right), \\
F_{J}=\frac{\left[(L+S+1)^{2}-J^{2}\right]\left[J^{2}-(S-L)^{2}\right]}{J} .
\end{gathered}
$$

Here, $k_{\mathrm{B}}$ is the Boltzmann constant, $N_{\mathrm{A}}$ Avogadro's number, $\mu_{\mathrm{B}}$ the Bohr magneton, and $g_{J}$ the Landé $\mathrm{g}$ factor. The best fit of Eq. (2) to the experimental data, which is represented by the red solid line in Fig. 1, gives the value of $\lambda=481 \mathrm{~K}$. This is in good agreement with that obtained from the theoretical calculation on the assumption of free $\operatorname{Eu}^{3+}(\lambda=460 \mathrm{~K})^{28)}$ and the previous experiment using a polycrystalline sample $\left.(\lambda \sim 476 \mathrm{~K}),{ }^{6}\right)$ indicating the pure $\mathrm{Eu}^{3+}$ state of $\mathrm{EuBe}_{13}$ at temperatures below $300 \mathrm{~K}$.

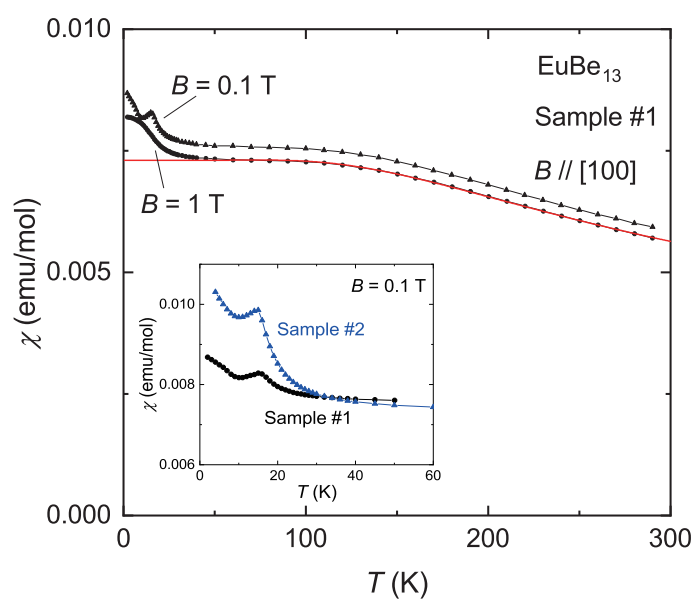

Fig. 1. (Color online) Temperature dependence of the magnetic susceptibility $\chi(T)$ for sample \#1 of $\operatorname{EuBe}_{13}$ at $B=0.1$ and $1 \mathrm{~T}(B / /[100])$. The red solid line represents the fitting curve on the basis of the Van Vleck susceptibility, as described in the text. The inset shows $\chi(T)$ below $60 \mathrm{~K}$ for samples $\# 1$ and $\# 2$ at $B=0.1 \mathrm{~T}$.

\subsection{Specific heat}

Figure 2 shows the temperature dependence of the specific heat divided by the temperature $C(T) / T$ for sample \#3 of $\mathrm{EuBe}_{13} . C(T) / T$ for $\mathrm{LaBe}_{13}$ is also displayed in this figure for comparison. ${ }^{19)}$ The $C(T) / T$ curve for $\mathrm{EuBe}_{13}$ is similar to that for $\mathrm{LaBe}_{13}$, indicating that the contribution of $4 f$ electrons of the Eu ion to the specific heat is negligibly small. In addition, there is no indication of a phase transition near 15 
$\mathrm{K}$, where the cusp anomaly was observed in the $\chi(T)$ curve. As shown in the inset of Fig. 2, the $C(T) / T$ curve obeys the Debye $T^{3}$ law below $\sim 13 \mathrm{~K}: C(T) / T=\gamma+\beta T^{2}$. Note that the present experimental data slightly deviates from the Debye $T^{3}$ law in the lowest-temperature region, which may be due to magnetic impurities as mentioned above. The Debye temperature $\theta_{\mathrm{D}}$ can be determined from the following expression:

$$
\theta_{\mathrm{D}}=\left(12 \pi^{4} R n / 5 \beta\right)^{1 / 3},
$$

where $R$ is the gas constant and $n(=14)$ is the number of atoms in the formula unit. From the experimental results, we determined $\gamma$ and $\theta_{\mathrm{D}}$ as $\sim 10.2 \mathrm{mJmol}^{-1} \mathrm{~K}^{-2}$ and $\sim 835 \mathrm{~K}$, respectively. These obtained values for $\mathrm{EuBe}_{13}$ are comparable to those reported for $\mathrm{LaBe}_{13}: \gamma \sim 9 \mathrm{mJmol}^{-1} \mathrm{~K}^{-2}$ and $\theta_{\mathrm{D}} \sim 750$ $-950 \mathrm{~K} .{ }^{6,19-21)}$

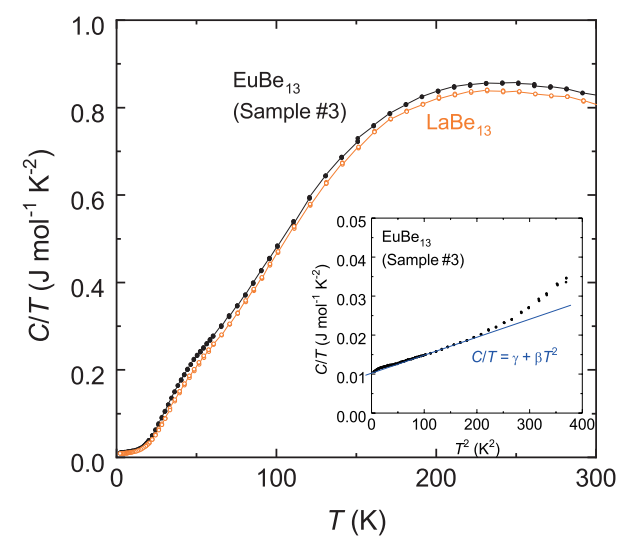

Fig. 2. (Color online) Temperature dependence of $C / T$ for $\mathrm{EuBe}_{13}$ (closed symbols) and $\mathrm{LaBe}_{13}$ (open symbols) ${ }^{19)}$ below $300 \mathrm{~K}$ at zero field. The inset shows the low-temperature region of $C(T) / T$ as a function of $T^{2}$. The blue line represents the Debye $T^{3}$ law.

For nonmagnetic $\mathrm{MBe}_{13}$ compounds, a hump structure is observed in $C(T) / T$ at approximately $35 \mathrm{~K},{ }^{19,29)}$ which can be regarded as the contribution of the low-energy Einstein phonon due to the oscillation of the $\mathrm{M}$ ion. To estimate the

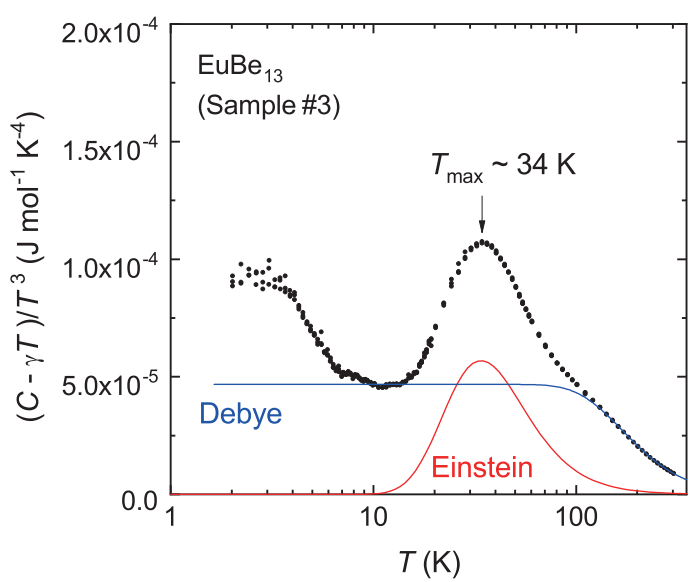

Fig. 3. (Color online) Temperature dependence of $(C-\gamma T) / T^{3}$ for $\mathrm{EuBe}_{13}$. The blue and red curves represent the Debye and Einstein phonon contribution calculated using the obtained $\gamma, \theta_{\mathrm{D}}$, and $\theta_{\mathrm{E}}$. contribution of the Einstein phonon, we plotted the temperature dependence of $(C-\gamma T) / T^{3}$ for $E \mathrm{BBe}_{13}$ as shown in Fig. 3 . The blue solid line represents the Debye specific heat calculated using the obtained $\gamma$ and $\theta_{\mathrm{D}}$. The deviation of $(C-$ $\gamma T) / T^{3}$ from the Debye specific heat below $10 \mathrm{~K}$ may be due to magnetic impurities. It is noteworthy that the $(C-\gamma T) / T^{3}$ curve shows a broad peak at $T_{\max } \sim 34 \mathrm{~K}$, which should originate from the contribution of the Einstein phonon. $T_{\max }$ is linked to $\theta_{\mathrm{E}}$ via the relationship $\theta_{\mathrm{E}} \sim 4.92 T_{\max },{ }^{11)}$ from which we estimated $\theta_{\mathrm{E}}$ for $\mathrm{EuBe}_{13}$ to be $\sim 167 \mathrm{~K}$. This estimated $\theta_{\mathrm{E}}$ value is fairly close to those reported previously for the other isostructural $\mathrm{MBe}_{13}$ compounds, $\mathrm{LaBe}_{13}, \mathrm{SmBe}_{13}$, $\mathrm{UBe}_{13}$, and $\mathrm{ThBe}_{13}{ }^{18-20)}$

\subsection{Electrical resistivity}

Figures 4(a) and 4(b) display the temperature dependence of the electrical resistivity $\rho(T)$ for $\mathrm{EuBe}_{13}$ measured on sample \#1 and $\mathrm{LaBe}_{13}$ taken from Ref. 19, respectively. The electrical current $I$ was applied along the [100] direction in both the measurements. The $\rho(T)$ curve for $\mathrm{EuBe}_{13}$ exhibits simple metallic behavior and is similar to that for $\mathrm{LaBe}_{13}$, indicating that $\mathrm{EuBe}_{13}$ is a nonmagnetic metallic compound with $\mathrm{Eu}^{3+}$. $\rho(T)$ for $\mathrm{EuBe}_{13}$ shows $T^{3}$-like behavior at low temperatures; neither $T^{5}$ due to the electron-Debye phonon scattering nor $T^{2}$ due to the electron-electron scattering, as shown in the inset of Fig. 4(a). Intriguingly, such $T^{3}$-like dependence was also observed in $\rho(T)$ for $\mathrm{LaBe}_{13}$ [see the inset of Fig. 4(b)]. These findings suggest that this unusual temperature dependence is a common feature for nonmagnetic $\mathrm{MBe}_{13}$ systems; that is, it originates from the electron-phonon scattering due to the presence of the low-energy phonon mode with $\theta_{\mathrm{E}} \sim 160$ K.

\section{Discussion}

We now consider the following model on the basis of Matthiessen's rule to explain the $\rho(T)$ curves for $\mathrm{EuBe}_{13}$ and $\operatorname{LaBe}_{13}: \rho_{\text {Total }}(T)=\rho_{0}+\rho_{\text {Deb }}(T)+\rho_{\text {Ein }}(T)$. The electronelectron scattering can be ignored since $T^{2}$ behavior is not observed within the experimental accuracy. In this formula, $\rho_{0}$ is the residual resistivity, $\rho_{\text {Deb }}$ is the Debye phonon contribution to $\rho$ described by the Bloch-Grüneisen law written $\operatorname{as}^{30,31)}$

$$
\begin{gathered}
\rho_{\text {Deb }}(T)=A_{\operatorname{Deb}}\left(\frac{T}{\theta_{\mathrm{D}}}\right)^{5} \int_{0}^{\theta_{\mathrm{D}} / T} \frac{x^{5}}{[\exp (x)-1][1-\exp (-x)]} d x \\
A_{\text {Deb }}=\frac{C}{m \theta_{\mathrm{D}}}
\end{gathered}
$$

and $\rho_{\text {Ein }}$ is the Einstein phonon contribution to the resistivity written as $^{32)}$

$$
\begin{gathered}
\rho_{\text {Ein }}(T)=\frac{A_{\text {Ein }}}{T\left[\exp \left(\frac{T}{\theta_{\mathrm{E}}}\right)-1\right]\left(\left[1-\exp \left(-\frac{T}{\theta_{\mathrm{E}}}\right)\right]\right.}, \\
A_{\text {Ein }}=\frac{K N}{m} .
\end{gathered}
$$

Here, $C$ is a constant, which is independent of the kind of material, $m$ the mass of an oscillator, $N$ the number of oscillators per unit cell volume, and $K$ a constant, which depends on the electron density of the metal and the electron-local-mode coupling strength. 
The $\rho(T)$ curves for $\mathrm{EuBe}_{13}$ and $\mathrm{LaBe}_{13}$ were analyzed using the above formula of $\rho_{\text {Total }}(T)$. In a fitting using $\rho_{0}, A_{\text {Deb }}$, $\theta_{\mathrm{D}}, A_{\mathrm{Ein}}$, and $\theta_{\mathrm{E}}$ as free parameters, we were unable to determine the values of these parameters uniquely, because the obtained values after the fitting depend on the initial parameters. Hence, in the present analyses, we fixed $\theta_{\mathrm{D}}$ and $\theta_{\mathrm{E}}$ to the values determined in other experiments. In the case of $\mathrm{EuBe}_{13}$, the values of $\theta_{\mathrm{D}}$ and $\theta_{\mathrm{E}}$ were fixed to those obtained from the present $C$ measurements: $\left(\theta_{\mathrm{D}}, \theta_{\mathrm{E}}\right)=(835 \mathrm{~K}, 167 \mathrm{~K})$. The fixed and obtained fitting parameters are summarized in Table I. The calculated $\rho(T)$ curve reproduces the experimental data reasonably well, as shown Fig. 4(a), where the calculated $\rho_{\text {Deb }}$ and $\rho_{\text {Ein }}$ are also shown. On the other hand, for $\mathrm{LaBe}_{13}$, we performed the analysis using two sets of $\theta_{\mathrm{D}}$ and $\theta_{\mathrm{E}}$ as the fixed parameters: $\left(\theta_{\mathrm{D}}, \theta_{\mathrm{E}}\right)=(920 \mathrm{~K}, 177 \mathrm{~K})$ obtained from the $C$ measurements by the authors' group ${ }^{19)}$ named Case 1 , and $\left(\theta_{\mathrm{D}}, \theta_{\mathrm{E}}\right)=(820 \mathrm{~K}, 163 \mathrm{~K})$ obtained from the $C$ measurements by Bucher et al. ${ }^{6}$ and the XRD measurements, ${ }^{20)}$ named Case 2 . In both cases, the $\rho_{\text {Total }}(T)$ curves appear to reproduce the experimental data in the main panel of Fig. 4(b). However, Case 2 gives a better description of $\rho(T)$ for $\mathrm{LaBe}_{13}$ than Case 1 since $\rho_{\text {Total }}(T)$ in Case 1 deviates from the experimental data in the $T^{3}$ plot [see the inset of Fig. 4(b)]. The deviation from the experimental data in Case 1 is considered to be due to the rather higher $\theta_{\mathrm{D}}$ and $\theta_{\mathrm{E}}$ than the typical values reported for the $\mathrm{MBe}_{13}$ compounds, ${ }^{18,20)}$ although it is unclear why our $C$ measurements for $\mathrm{LaBe}_{13}$ give higher values of $\theta_{\mathrm{D}}$ and $\theta_{\mathrm{E}} \cdot{ }^{19)}$

Here, we evaluate the obtained $A_{\mathrm{Deb}}$ and $A_{\text {Ein }}$ parameters (Table I) from the view point of the oscillators of the Debye and Einstein phonons in $\mathrm{MBe}_{13}$ systems. Using the $A_{\text {Deb }}$ parameters of $\mathrm{EuBe}_{13}$ and $\mathrm{LaBe}_{13}$ obtained from the present $\rho$ measurements, $A_{\mathrm{Deb}}^{\mathrm{EuBe}_{13}} / A_{\mathrm{Deb}}^{\mathrm{LaBe}_{13}}$ is estimated to be $\sim 1.05$. When the masses of the Debye oscillators are the same for $\mathrm{EuBe}_{13}$ and $\mathrm{LaBe}_{13}$, i.e., the $\mathrm{Be}$ atom is the Debye oscillator, $A_{\mathrm{Deb}}^{\mathrm{EuB}_{13}} / A_{\mathrm{Deb}}^{\mathrm{LaBe}_{13}}$ is calculated to be $\sim 0.98$ from Eq. (8) using $\theta_{\mathrm{D}}^{\mathrm{EuBe}_{13}}$ and $\theta_{\mathrm{D}}^{\mathrm{LaBe}_{13}}$ (Case 2). On the other hand, $A_{\mathrm{Deb}}^{\mathrm{EuBe}_{13}} / A_{\mathrm{Deb}}^{\mathrm{LaB}_{13}}$ becomes $\sim 0.90$ on the assumption that the Debye oscillators are the $\mathrm{Eu}$ and $\mathrm{La}$ ions, which is more distant from 1.05. Here, the atomic masses of $\mathrm{La}$ and $\mathrm{Eu}$ are 138.91 and 151.96, respectively. These results support the suggestion that the Be atoms form the crystal lattice described by the Debye model given by the previous powder XRD measurements of $\mathrm{MBe}_{13} \cdot{ }^{20)}$

For the Einstein phonon, $A_{\mathrm{Ein}}^{\mathrm{EuBe}_{13}} / A_{\mathrm{Ein}}^{\mathrm{LaBe}_{13}}$ can be rewritten as $m_{\text {Ein }}^{\mathrm{LaBe}_{13}} / m_{\text {Ein }}^{\mathrm{EuBe}_{13}}$ from Eq. (10) when $K$ and $N$ are the same for the two compounds. Here, $m_{\text {Ein }}$ is the mass of an Einstein oscillator. Since it has been revealed that the Einstein oscillator in the $\mathrm{MBe}_{13}$ compounds is the $\mathrm{M}$ atom, ${ }^{18,20)}$ the masses of $\mathrm{La}$ and $\mathrm{Eu}$ are adopted as $m_{\mathrm{Ein}}^{\mathrm{LaB}_{13}}$ and $m_{\mathrm{Ein}}^{\mathrm{EuBe}_{13}}$, respectively. The estimated $A_{\mathrm{Ein}^{\mathrm{EuB}}}^{\mathrm{Eu}_{13}} / A_{\mathrm{Ein}^{\mathrm{LaB}}}^{\mathrm{LaB}_{13}}(\sim 0.96)$ shows good agreement with $m_{\mathrm{Ein}}^{\mathrm{LaBe}_{13}} / m_{\mathrm{Ein}}^{\mathrm{EuBe}_{13}}(\sim 0.91)$. This result indicates the validity of the present assumption that the $\mathrm{M}$ atom is the Einstein oscillator of the low-energy phonon mode, although we cannot exclude the possibility that the Be atoms are the Einstein oscillators only from this analysis. This result also suggests that the conduction electron density and the electron-local-mode coupling of $\mathrm{EuBe}_{13}$ are similar to those of $\mathrm{LaBe}_{13}$, because we assumed the same value of $K$ for the two compounds in the present analysis. To investigate the systematic changes in the parameters of $\rho_{\text {Total }}(T)$ and whether the $T^{3}$-like behavior
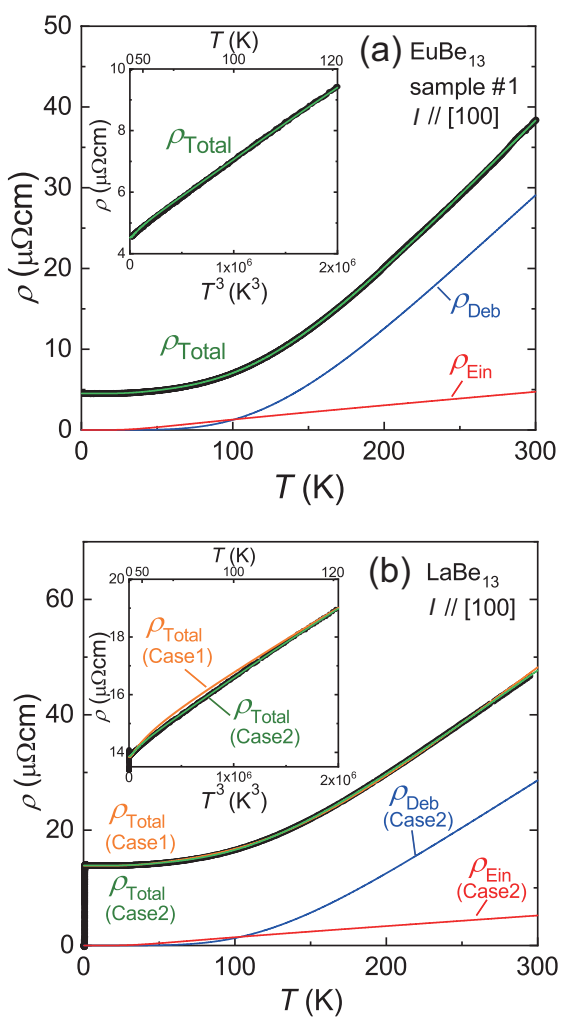

Fig. 4. (Color online) Temperature dependence of the electrical resistivity of (a) $\mathrm{EuBe}_{13}$ measured on sample \#1 and (b) $\mathrm{LaBe}_{13}$ taken from Ref. 19. The insets in both figures show the $\rho(T)$ data at low temperatures as a function of $T^{3}$. The green and orange solid curves represent the fitting curves of $\rho_{\text {Total }}$, as described in the text, while the blue and red solid curves represent the components of $\rho$ attributed to the Debye and Einstein phonon scattering ( $\rho_{\text {Deb }}$ and $\left.\rho_{\text {Ein }}\right)$, respectively.

in $\rho(T)$ are common to the $\mathrm{MBe}_{13}$ systems, further studies are required for other $\mathrm{MBe}_{13}$ compounds with the nonmagnetic ground state, such as $\mathrm{LuBe}_{13}$ and $\mathrm{ThBe}_{13}$, are now in progress.

Finally we comment on the valence state of the Eu ion in $\mathrm{EuBe}_{13}$. The valence state of the Eu ion has a strong correlation with the lattice constant because the ionic radius of $\mathrm{Eu}^{2+}$ is larger than that of $\mathrm{Eu}^{3+}{ }^{33)}$ In this paper, the free distance of the Eu ion in the material is considered for the comparison among different types of Eu-based compounds. Here, the free distance is defined as $\delta r\left(=r_{\mathrm{Eu}-\mathrm{FNN}}-r_{\mathrm{FNN}}\right)$, where $r_{\mathrm{Eu}-\mathrm{FNN}}$ is the distance between $\mathrm{Eu}$ and the first-nearest-neighbour atom $(\mathrm{FNN})$, and $r_{\mathrm{FNN}}$ is adopted to be the covalent atomic radius of the FNN. ${ }^{34)}$ Figure 5 displays the Eu valence states plotted against $\delta r$ in various Eu-based cubic intermetallics. ${ }^{35-43)}$ The red and blue solid lines represent the effective ionic radii of $\mathrm{Eu}^{2+}\left(r_{\mathrm{Eu}}^{2+}=1.30 \mathrm{~A}\right)$ and $\mathrm{Eu}^{3+}\left(r_{\mathrm{Eu}}^{3+}=1.12 \mathrm{~A}\right)$ for the 9coordination-number site, respectively. ${ }^{33)}$ As seen in Fig. 5, a boundary between $\mathrm{Eu}^{2+}$ and $\mathrm{Eu}^{3+}$ in these materials appears to be present at $\delta r \sim 1.8 \AA$, in other words, there is a tendency for the larger free space to stabilize the $\mathrm{Eu}^{2+}$ state. Intriguingly, only $\mathrm{EuBe}_{13}$ does not follow this tendency. Thus, $\mathrm{EuBe}_{13}$ is found to be a unique Eu-based intermetallic with the trivalent state in spite of the large free distance.

The unique $\mathrm{Eu}^{3+}$ state in $\mathrm{EuBe}_{13}$ might originate from the characteristic ligands forming the Be-caged structure rather than from the free distance, since the rare-earth $\mathrm{MBe}_{13}$ compounds commonly have the trivalent state even in $\mathrm{SmBe}_{13}$ and 
Table I. Fixed parameters of Debye temperature $\theta_{\mathrm{D}}$, and Einstein temperature $\theta_{\mathrm{E}}$, and fitting parameters of $A_{\text {Deb }}, A_{\text {Ein }}$, and $\rho_{0}$ in the present model calculation for describing $\rho(T)$ of $\mathrm{EuBe}_{13}$ and $\mathrm{LaBe}_{13} . \theta_{\mathrm{D}}$ and $\theta_{\mathrm{E}}$ were taken from the present $C$ measurement and the literature. $\left.6,19,20\right)$

\begin{tabular}{lcc:ccc}
\hline \hline & $\theta_{\mathrm{D}}(\mathrm{K})$ & $\theta_{\mathrm{E}}(\mathrm{K})$ & $A_{\text {Deb }}\left(\mu \Omega \mathrm{cm} \mathrm{K}^{-1}\right)$ & $A_{\text {Ein }}\left(\mu \Omega \mathrm{cm} \mathrm{K} \mathrm{K}^{-1}\right)$ & $\rho_{0}(\mu \Omega \mathrm{cm})$ \\
\hline $\mathrm{EuBe}_{13}$ & $835^{\mathrm{a})}$ & $167^{\mathrm{a})}$ & 483 & 451 & 4.52 \\
$\mathrm{LaBe}_{13}$ (Case 1) & $920^{\mathrm{b})}$ & $177^{\mathrm{b})}$ & 531 & 819 & 13.84 \\
$\mathrm{LaBe}_{13}$ (Case 2) & $820^{\mathrm{c})}$ & $163^{\mathrm{d})}$ & 461 & 472 & 13.86 \\
\hline
\end{tabular}

\footnotetext{
a) Specific Heat (Present Study)

b) Specific Heat (Hidaka et al.) ${ }^{[19]}$

c) Specific Heat (Bucher et al.) ${ }^{[6]}$

d) Powder XRD (Hidaka et al.) [20]
}

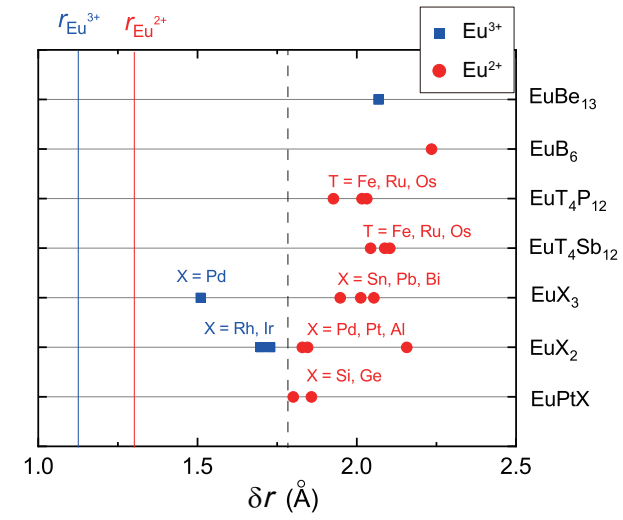

Fig. 5. (Color online) Eu valence states in various Eu-based cubic compounds (circles, $\mathrm{Eu}^{2+}$; squares, $\mathrm{Eu}^{3+}$ ). $\delta r$ is a measure of the free distance of the Eu ion. The red and blue solid lines represent the effective ionic radii of $\mathrm{Eu}^{2+}$ and $\mathrm{Eu}^{3+}$, respectively. ${ }^{33)}$

$\mathrm{YbBe}_{13},{ }^{6,44-46)}$ except for $\mathrm{CeBe}_{13} .{ }^{3,47)}$ One possible explanation is that the ionic radius of the Eu ion is effectively enlarged owing to the low-energy phonon mode with the large local oscillation. However, this possibility appears to be unlikely because $\mathrm{EuB}_{6}$ and $\mathrm{EuT}_{4} \mathrm{Sb}_{12}(\mathrm{~T}=$ transition metals) also have a low-energy phonon mode, whose $\theta_{\mathrm{E}}$ are close to that in $\mathrm{EuBe}_{13} \cdot{ }^{35,48)}$ Another possibility is that the energy loss due to the lattice expansion defeats the energy gain by taking the $\mathrm{Eu}^{2+}$ state with the larger ionic radius, even though the present compound has enough free space. Since the $\mathrm{MBe}_{13}$ compounds have a rigid Be host cage with a high $\theta_{\mathrm{D}}$ of $\sim$ $800 \mathrm{~K},{ }^{20,21)}$ the lattice expansion might induce a large energy loss, even for a tiny expansion. For comparison, we enumerate $\theta_{\mathrm{D}}$ for several La-substituted compounds instead of those for the Eu-based compounds shown in Fig. 5: $\theta_{\mathrm{D}}=262 \mathrm{~K}$ for $\mathrm{LaRu}_{4} \mathrm{Sb}_{12},{ }^{35)} 304 \mathrm{~K}$ for $\mathrm{LaOs}_{4} \mathrm{Sb}_{12},{ }^{35)} 176 \mathrm{~K}$ for $\mathrm{LaPd}_{3},{ }^{49)}$ $205 \mathrm{~K}$ for $\mathrm{LaSn}_{3},{ }^{50)} 214 \mathrm{~K}$ for $\mathrm{LaRh}_{2},{ }^{51)} 352 \mathrm{~K}$ for $\mathrm{LaAl}_{2},{ }^{52}$ ) and $212-885 \mathrm{~K}$ for $\mathrm{LaB}_{6} .{ }^{53)}$ Elucidating the origin of the unique trivalent state of $\mathrm{EuBe}_{13}$ may provide further insights into not only the $f$ electronic properties in the $\mathrm{MBe}_{13}$ systems, including electron-phonon coupling, but also the valence instability in the Eu-based intermetallics.

\section{Summary}

We have succeeded in growing single crystals of $\mathrm{EuBe}_{13}$. We performed $\chi(T), C(T)$, and $\rho(T)$ measurements on them to investigate the Eu valence state and phonon properties. The pure $\mathrm{Eu}^{3+}$ state in $\mathrm{EuBe}_{13}$ was confirmed by an analysis of $\chi(T)$ on the basis of the Van Vleck theory. The contribution of the $4 f$ electrons to $C$ and $\rho$ are negligible below $300 \mathrm{~K}$, and the $C(T) / T$ and $\rho(T)$ curves can be explained well by a combination of the Debye phonon with $\theta_{\mathrm{D}} \sim 835 \mathrm{~K}$ and the Einstein phonon with $\theta_{\mathrm{E}} \sim 167 \mathrm{~K}$. These results indicate that $\mathrm{EuBe}_{13}$ is a new member of the $\mathrm{MBe}_{13}$ family showing a low-energy phonon mode. Furthermore, it is also revealed that the present compound takes an interesting position among the Eu-based cubic compounds with respect to the relationship between the $\mathrm{Eu}$ valence and free distance of the Eu ion.

The authors thank Dr. C. Tabata and Dr. Y. Shimizu for fruitful discussions. The present research was supported by JSPS KAKENHI Grants No. JP20224015(S), No. JP25400346(C), No. JP26400342(C), No. JP15H05882, and No. JP15H05885(J-Physics).

1) H. R. Ott, H. Rudigier, Z. Fisk, and J. L. Smith, Phys. Rev. Lett. 50, 1595 (1983).

2) H. M. Mayer, U. Rauchschwalbe, C. D. Bredl, H. Rietshel, H. Schmidt, H. Wühl, and J. Beuers, Phys. Rev. B 33, 3168 (1986).

3) Z. S. Wilson, R. T. Macaluso, E. D. Bauer, J. L. Smith, J. D. Thompson, Z. Fisk, G. G. Stanley, and J. Y. Chan, J. Am. Chem. Soc. 126, 13926 (2004).

4) F. Bourée-Vigneron, Phys. Scr. 44, 27 (1991).

5) P. L. Moyland, T. Lang, E. D. Adams, G. R. Stewart, and Y. Takano, Czech. J. Phys. 46, Suppl. S4, 2199 (1996).

6) E. Bucher, J. P. Maita, G. W. Hull, R. C. Fulton, and A. S. Cooper, Phys. Rev. B 11, 440 (1975).

7) M. W. McElfresh, J. H. Hall, R. R. Ryan, J. L. Smith, and Z. Fisk, Acta Crystallogr. C46, 1579 (1990).

8) K. Takegahara, H. Harima, and T. Kasuya, J. Phys. F: Met. Phys. 16, 1691 (1986).

9) A. D. Caplin and G. Grüner, Phys. Rev. Lett. 30, 1138 (1973).

10) D. Mandrus, B. C. Sales, and R. Jin, Phys. Rev. B 64, 012302 (2001).

11) K. Matsuhira, Y. Hinatsu, C. Sekine, T. Togashi, H. Maki, I. Shirotani, H. Kitazawa, T. Takamasu, and G. Kido, J. Phys. Soc. Jpn. 71, Suppl. 237 (2002).

12) J.-I. Yamaura, S. Yonezawa, Y. Muraoka, and Z. Hiroi, J. Solid State 
Chem. 179, 336 (2006).

13) K. Suekuni, M. A. Avila, K. Umeo, H. Fukuoka, S. Yamanaka, T. Nakagawa, and T. Takabatake, Phys. Rev. B 77, 235119 (2008).

14) J.-I. Yamaura and Z. Hiroi, J. Phys. Soc. Jpn. 80, 054601 (2011).

15) Y. Nagao, J.-I. Yamaura, H. Ogusu, Y. Okamoto, and Z. Hiroi, J. Phys. Soc. Jpn. 78, 064702 (2009).

16) S. Sanada, Y. Aoki, H. Aoki, A. Tsuchiya, D. Kikuchi, H. Sugawara, and H. Sato, J. Phys. Soc. Jpn. 74, 246 (2005).

17) K. Hattori, Y. Hirayama, and K. Miyake, J. Phys. Soc. Jpn. 74, 3306 (2005).

18) B. Renker, F. Gompf, W. Reichardt, H. Rietschel, and J. B. Suck, and J. Beuers, Phys. Rev. B 32, 1859 (1985).

19) H. Hidaka, Y. Shimuzu, S. Yamazaki, N. Miura, R. Nagata, C. Tabata, S. Mombetsu, T. Yanagisawa, and H. Amitsuka, J. Phys. Soc. Jpn. 80, 043601 (2017).

20) H. Hidaka, R. Nagata, C. Tabata, Y. Shimuzu, N. Miura, T. Yanagisawa, and H. Amitsuka, Phys. Rev. Mater. 2, 053603 (2018).

21) J. P. Kappler, G. Krill, M. F. Ravet, G. Heinrich, and A. Meyer, J. Magn. Magn. Mater. 15-18, 965 (1980).

22) For experimental reviews, see, for example, Y. Ōnuki, A. Nakamura, F. Honda, D. Aoki, T. Takeuchi, M. Nakashima, Y. Amako, H. Harima, K. Matsubayashi, Y. Uwatoko, S. Kayama, T. Kagayama, K. Shimizu, S. E. Muthu, D. Braithwaite, B. Salce, H. Shiba, T. Yara, Y. Ashitomi, H. Akamine, K. Tomori, M. Hedo, and T. Nakama, Philos. Mag. 97, 3399 (2016).

23) T. Hotta, J. Phys. Soc. Jpn. 84, 114707 (2015).

24) I. Nowik and I. Felner, Hyperfine Interact. 28, 959 (1986).

25) J. M. Bloch, D. Davidov, I. Felner, and D. Shaltiel, J. Phys. F: Met. Phys. 6, 1979 (1976).

26) A. Nakamura, T. Uejo, F. Honda, T. Takeuchi, H. Harima, E. Yamamoto, Y. Haga, K. Matsubayashi, Y. Uwatoko, M. Hedo, T. Nakama, and Y. Ōnuki, J. Phys. Soc. Jpn. 84, 124711 (2015).

27) J. H. Van Vleck, The Theory of Electric and Magnetic S usceptibilities (Oxford University Press, 1932).

28) E. R. Judd, Proc. Phys. Soc. A69, 157 (1956).

29) R. Felten, F. Steglich, G. Weber, H. Rietscel, F. Gompf, B. Renker, and J. Beuers, Europhys. Lett. 2, 321 (1986).

30) J. M. Ziman. Electrons and Phonons: The Theory of Transport Phenomena in Solids (Oxford University Press, 1960).

31) K. Ueda and Y. Ōnuki, Physics of Heavy Electron Systems (Shokabo, Tokyo, 1998).

32) J. R. Cooper, Phys. Rev. B 9, 2778 (1974).

33) R. D. Shannon, Acta Crystallogr. A32, 751 (1976).

34) B. Cordero, V. Gómez, A. E. Platero-Prats, M. Revés, J. Echeverría, E. Cremades, F. Barragán, and S. Alvarez, Dalton Trans., 2832 (2008).

35) E. D. Bauer, A. Ślebarski, N. A. Frederick, W. M. Yuhasz, M. B. Maple,
D. Cao, F. Bridges, G. Giester, and P. Rogl, J. Phys: Condens. Matter 16, 5095 (2004).

36) S. Süllow, I. Prasad, M. C. Aronson, J. L. Sarrao, Z. Fisk, D. Hristova, A. H. Lecerda, M. F. Hundley, A. Vigliante, and D. Gibbs, Phys. Rev. B 57, 5860 (1998).

37) F. Grandjean, A. Gerard, J. Hodges, D. J. Braun, and W. Jeitschko, Hyperfine Interact. 15/16, 765 (1983).

38) C. Sekine, M. Inoue, T. Inaba, and I. Shirotani, Physica B 281\&282, 308 (2000).

39) K. Kihou, I. Shirotani, Y. Shimaya, C. Sekine, and T. Yagi, Mater. Res. Bull. 39, 317 (2004).

40) A. Nakamura, H. Akamine, Y. Ashitomi, F. Honda, D. Aoki, T. Takeushi, K. Matsubayashi, Y. Uwatoko, Y. Tatetsu, T. Maehira, M. Hedo, T. Nakama, and Y. Ōnuki, J. Phys. Soc. Jpn. 85, 084705 (2016).

41) M. Kakihana, K. Nishimura, Y. Ashitomi, T. Yara, D. Aoki, A. Nakamura, F. Honda, M. Nakashima, Y. Amako, Y. Uwatoko, T. Sakakibara, S. Nakamura, T. Takeuchi, Y. Haga, E. Yamamoto, H. Harima, M. Hedo, T. Nakama, and Y. Ōnuki, J. Electron Mater. 46, 3572 (2017).

42) M. Kakihana, D. Aoki, A. Nakamura, F. Honda, M. Nakashima, Y. Amako, S. Nakamura, T. Sakakibara, M. Hedo, T. Nakama, and Y. Ōnuki, J. Phys. Soc. Jpn. 87, 023701 (2018).

43) R. Pöttgen, R. K. Kramer, W. Schnelle, R. Müllmann, and B. D. Mosel, J. Mater. Chem. 6, 635 (1996).

44) H. Hidaka, S. Yamazaki, Y. Shimuzu, N. Miura, C. Tabata, T. Yanagisawa, and H. Amitsuka, J. Phys. Soc. Jpn. 86, 074703 (2017).

45) S. Tsutsui, R. Masuda, Y. Kobayashi, Y. Yoda, K. Mizuuchi, Y. Shimizu, H. Hidaka, T. Yanagisawa, H. Amitsuka, F. Iga, and M. Seto, J. Phys. Soc.

Jpn. 85, 083704 (2016)

46) G. Heinrich, J. P. Kappler, and A. Meyer, Phys. Lett. 74A, 121 (1979).

47) D. Lenz, H. Schmidt, S. Ewert, W. Boksch, R. Pott, and D. Wohlleben, Solid State Commun. 52, 759 (1984).

48) Y. Takahashi, K-I. Ohshima, F. P. Okamura, S. Otani, T. Tanaka, and J. M. Lawrence, J. Phys. Soc. Jpn. 68, 2304 (1999).

49) J. M. Machado da Silva, Solid State Commun. 28, 857 (1978).

50) G. A. Costa, F. Canepa, and G. L. Olcese, Solid State Commun. 40, 169 (1981).

51) H. Sugawara, T. Yamazaki, J. Itoh, M. Takashita, T. Ebihara, N. Kimura, P. Svoboda, R. Settai, Y. Ōnuki, H. Aoki, and S. Uji, Physica B 199\&200, 570 (1994).

52) R. E. Hungsberg and K. A. Gschneidner Jr., J. Phys. Chem. Solids 33, 401 (1972).

53) M. M. Korsukova, T. Lundström, L. E. Tergenius, and V. N. Gurin, Solid State Commun. 63, 187 (1987). 